\title{
Análise da resposta ao biofeedback nos pacientes com incontinência fecal
}

\author{
Analysis of biofeedback for fecal incontinence
}

\author{
ANDRÉ FIGUEIREDO ACCETTA ${ }^{1}$, RICARDO S. VASCONCELOS², GISELE DEL CUETO ${ }^{3}$, JOÃO DE A. PUPO \\ $\mathrm{NETO}^{4}$, DOMINGOS LACOMBE${ }^{4}$, ITALO ACCETTA ${ }^{5}$
}

\begin{abstract}
${ }^{1}$ Professor de Cirurgia da Faculdade de Medicina DA Universidade Federal Fluminense (UFF) - Niterói (RJ), Brasil. ${ }^{2}$ Médico Coloproctologista do Hospital Universitário Clementino Fraga Filho (HUCFF) - Rio de Janeiro (RJ), Brasil. ${ }^{3}$ Médica Coloproctologista do Hospital Municipal Miguel Couto - Rio de Janeiro (RJ), Brasil. ${ }^{4}$ Professor Adjunto de Coloproctologia da Faculdade de Medicina da Universidade Federal do Rio de Janeiro (UFRJ) - Rio de Janeiro (RJ), Brasil. ${ }^{5}$ Professor Titular de Cirurgia da Faculdade de Medicina da Universidade Federal Fluminense (UFF); Professor da Universidade Gama Filho - Rio de Janeiro (RJ), Brasil.
\end{abstract}

ACCETTA AF, VASCONCELOS RS, CUETO GD, NETO JDAP, LACOMBE D, ACCETTA I. Análise da resposta ao biofeedback nos pacientes com incontinência fecal. Rev bras Coloproct, 2011;31(2): 165-168.

RESUMO: Incontinência fecal é uma condição com importante impacto na qualidade de vida, e inúmeras formas de tratamento são descritas. Objetivo: Avaliar a resposta ao tratamento por biofeedback e o perfil epidemiológico dos pacientes com incontinência fecal, descrevendo os critérios de seleção e a técnica utilizada. Métodos: Estudo retrospectivo dos pacientes tratados em três anos (junho de 2005 a junho de 2008). Resultados: Trinta pacientes, sendo 26 mulheres e 4 homens, com idade média de 66 anos. $O$ número de gestações e partos normais variou de nenhuma a seis e a histerectomia esteve presente em nove casos. Todos os pacientes apresentavam hipotonia na manometria. Dezoito pacientes ficaram satisfeitos com o tratamento proposto, dez ficaram parcialmente satisfeitos, nenhum ficou completamente insatisfeito, e dois abandonaram a terapia. Conclusão: $O$ tratamento clínico associado ao biofeedback pode ser eficaz para a melhoria dos sintomas; entretanto, o entendimento e compreensão do problema por parte do paciente parece ser o efeito mais importante para esses resultados. A presença de diabetes mellitus, cirurgias orificiais e histerectomia podem ter relação com as queixas de incontinência.

Palavras-chave: biofeedback; incontinência fecal; exercício; manometria; qualidade de vida.

\section{INTRODUÇÃO}

Incontinência fecal é uma condição com relevante custo social. Atinge principalmente pessoas idosas ${ }^{1}$, e com o envelhecimento da população, está cada vez mais presente nos consultórios de coloproctologia. Possui etilogia multifatorial, razão pela qual não existe um tratamento universal, devendo ser individualizado para cada caso. Inúmeras formas de terapia são descritas, que variam desde a medidas higieno-dietéticas, drogas constipantes, até cirurgias complexas. Avaliar a resposta ao biofeedback e o perfil epidemiológico dos pacientes com incontinência fecal foi o principal objetivo deste trabalho.

\section{PACIENTES E MÉTODOS}

Entre junho de 2005 a junho de 2008, 30 pacientes com queixas de incontinência fecal foram submetidos, por nós, a tratamento com biofeedback. Em todos, foi realizado estudo manométrico complementado com exames de imagem (ressonância magnética e ultrassom endoanal), quando necessários. $\mathrm{O}$ manômetro utilizado foi o Viotti 6600 de fluxo contí-

Trabalho realizado no Hospital Universitário Clementino Fraga Filho (HUCFF) - Rio de Janeiro (RJ), Brasil.

Fonte de financiamento: não há

Conflito de interesse: nada a declarar

Recebido em: 19/12/2008

Aprovado em: 09/03/2009 
nuo com quatro canais. $\mathrm{O}$ tratamento teve duração de três semanas e, após o período, foi avaliada a resposta através de um questionário subjetivo, para definir o grau de satisfação e da qualidade de vida. Também foi realizado um follow up com quatro meses, através de contato telefônico.

Pacientes com quadro de importante déficit neurológico pós-traumatismo crânio-encefálico, traumatismo raquimedular ou acidente vascular encefálico e hipotonia total com ânus entreaberto foram excluídos da presente série. A ruptura esfincteriana, passível de correção, também foi critério de exclusão, pois acreditamos que o biofeedbak não traria nenhum benefício e poderia postergar o tratamento definitivo. Pacientes que não compreenderam a dinâmica da terapia, também não foram submetidos ao biofeedback.

\section{Técnica}

Nossa técnica constou de uma consulta semanal durante três semanas, na qual eram explicados os métodos e realizados exames físicos e manométricos. $\mathrm{Na}$ primeira consulta, a terapia era explicada detalhadamente, bem como a fisiologia da evacuação e a importância da colaboração e cooperação para o sucesso do tratamento.

Em todos os pacientes foi associado tratamento clínico com fibras ( $30 \mathrm{~g} / \mathrm{dia})$ e loperamida $(2 \mathrm{mg} / \mathrm{dia})$. A proposta era que os exercícios fossem feitos duas vezes por dia no domicílio. Houve livre acesso ao médico assistente, através de contato pessoal ou telefônico para esclarecimento de dúvidas. Os pacientes também eram orientados a fazer anotação diária da alimentação, a fim de detectar possíveis alimentos causadores de escape.

Didaticamente, os exercícios eram divididos em três etapas: a primeira tratava-se na identificação da musculatura adequada. A segunda, fase de contração rápida, 15 contrações com intervalos de 5 segundos eram realizadas. Um descanso de 3 minutos era proposto antes da última etapa, a fase de contração lenta, na qual 15 longas e mantidas contrações eram executadas.

Durante todo o processo, o paciente acompanhava on time suas curvas de pressão no monitor, através de softwares ilustrativos. Nas outras semanas, as séries eram repetidas com distensão da ampola retal, com o volume encontrado no theshold.

\section{RESULTADOS}

O grupo estudado foi constituído por $26(86 \%)$ pacientes do sexo feminino e 4 (14\%) do sexo masculino, com idade média de 66 anos (37/84). Cinco (16\%) pacientes eram hipertensos, 4 (13\%) eram diabéticos e $3(10 \%)$ apresentavam essas duas comorbidades. Dois $(6 \%)$ pacientes apresentavam incontinência urinária associada à fecal, e dois $(6 \%)$, tratavam doença inflamatória intestinal, um doença de Crohn e outro retocolite ulcerativa. Nas mulheres, o números de gestações e partos normais variou de 0 a 6 . Nove $(30 \%)$ pacientes apresentavam alguma cirurgia orificial prévia, sendo que a hemorroidectomia esteve presente em $6(20 \%)$ casos. Nove mulheres (34\%) eram histerectomizadas. Todos os pacientes apresentavam algum grau de hipotonia esfincteriana comprovada na manometria.

Dezoito $(60 \%)$ pacientes ficaram satisfeitos com o tratamento proposto, dez (34\%) ficaram parcialmente satisfeitos, nenhum ficou completamente insatisfeito, e dois (6\%) abandonaram o tratamento.

\section{DISCUSSÃO}

Incontinência fecal é definida como perda recorrente e incontrolável de material fecal ${ }^{2}$. As principais causas são as anormalidades da mobilidade intestinal (diarreia ou constipação), alteração na sensibilidade e baixa complacência retal, fraqueza ou dano da musculatura pélvica, ou uma combinação desses fatores ${ }^{2,3}$.

Sua incidência varia de 2 a $7 \%$ na população adulta $^{2}$; porém acredita-se que esse número seja maior, pois muitos pacientes não procuram ajuda profissio$\mathrm{nal}^{4}$. É mais frequente em mulheres, principalmente após traumas obstétricos, onde dois mecanismos de injúria são descritos: a destruição direta do esfíncter anal e a neuropatia degenerativa do nervo pudendo ${ }^{4,5}$. Não há relato de mortalidade atribuída a incontinência fecal; entretanto, existe morbidade significativa nas doenças de pele, infecções urinárias, além do impacto na qualidade de vida ${ }^{2}$.

Biofeedback é relatado como efetivo para tratamento da incontinência fecal há vários anos ${ }^{2}$. Foi inicialmente descrito na década de 1970, como forma 
de melhorar os resultados dos programas de exercícios pélvicos ${ }^{4}$.

É definido como um conjunto de exercícios da musculatura anal e perineal, medidas educativas, orientação alimentar, social e medicamentosa, que visam melhorar a habilidade voluntária do esfíncter anal externo e puboretal, em resposta a distensão retal $^{6}$. É realizado sob orientação médica ambulatorial e domiciliar. Já foi diversas vezes investigado; entretanto, devido aos múltiplos protocolos existentes, diversos critérios de seleção e técnicas utilizadas, é difícil comparar os estudos de maneira universal ${ }^{7,8}$. Dessa maneira, seu mecanismo exato de ação permanece obscuro 9 .

Didaticamente, atua de três formas: na coordenação motora, na força muscular e na sensibilidade retal $^{6}$. Para Chiarioni et al. ${ }^{10}$ a principal disfunção nos pacientes com urgência e escape para líquidos é corrigida com a melhoria da força muscular. Em contraste, a incontinência para sólidos é melhor controlada com o treinamento da sensibilidade retal ${ }^{11}$.

É evidente que cada tipo de incontinência tem uma causa de disfunção, devendo o protocolo de tratamento ser individualizado para cada caso $^{6}$. Infelizmente, poucas vezes detectamos uma causa específica da incontinência, o que dificulta estabelecer uma terapia padrão.

O efeito feedback ocorre quando o paciente entende e compreende a fisiopatologia do seu tipo de incontinência. Dessa maneira cria-se uma cadeia de retroalimentação com o médico assistente e com o tratamento, o que colabora para um melhor resultado. Acreditamos que a visualização de figuras análogas ao esfíncter anal ou a gráficos de pressão no monitor, durante os exercícios, auxiliam a criação do efeito feedback. Entretanto, muitos autores acreditam que algum efeito placebo também pode ocorrer ${ }^{7,8}$.

É consenso que o uso de exames mais sofisticados, não substitui um bom exame clínico com toque retal ${ }^{1}$. No nosso trabalho, apenas a manometria foi indicada de rotina. Exames como ultrassonografia endoanal e ressonância nuclear magnética só foram solicitados para esclarecimento diagnóstico, a fim de descartar lesões passíveis de correção cirúrgica. O tempo de latência do pudendo também não foi solicitado, pois acreditamos que ele não serve para triar candidatos ao biofeedback.
Diversos autores tiveram bons resultados combinando o biofeedback com outros métodos de reabilitação, como fisioterapia e eletroestimulação 7 . Fynes et al. ${ }^{5}$, compararam o biofeedback convencional associado à estimulação elétrica no esfíncter anal com eletromiografia audiovisual pós-trauma obstétrico, com bons índices de satisfação.

Byrne et al. ${ }^{1}$ realizaram consultas de biofeedback por telefone para os pacientes que tinham dificuldade de ir para o hospital, obtendo resultados semelhantes aos da literatura, e ainda sugeriu uma redução do regime de tratamento.

Critérios subjetivos e objetivos após tratamento com biofeedback são amplamente discutidos. Diversos artigos mostram discrepância entre esses resultados, o que reflete a etiologia multifatorial e até mesmo psíquica da incontinência fecal ${ }^{12,13,14}$. Também é controverso realizar exames manométricos após o tratamento ${ }^{6}$. No nosso trabalho, apenas critérios subjetivos foram avaliados, e não foi realizada manometria de controle. Acreditamos que o mais importante, após o biofeedback, é a melhoria da qualidade de vida e o retorno das atividades habituais, mesmo ainda havendo hipotonia esfincteriana e escape eventual.

Algumas revisões concluem que o biofeedback é o tratamento de primeira linha para a incontinência fecal ${ }^{4,9}$. Entretanto, ele não substitui, nem posterga, o tratamento cirúrgico. Pode ser usado como tratamento adjuvante, melhorando os resultados das esfincteroplastias $^{5,6}$.

\section{CONCLUSÃO}

O tratamento clínico associado ao biofeedba$c k$ pode ser eficaz para a melhoria dos sintomas e da qualidade de vida dos pacientes com queixas de incontinência fecal. Os resultados foram semelhantes ao da literatura consultada. Acreditamos que o entendimento e compreensão do problema, por parte do paciente, parece ser o efeito mais importante para esses resultados.

A presença de diabetes mellitus, cirurgias orificiais e histerectomia tiveram relação com as queixas de incontinência; entretanto, novos estudos com maior casuística e análise estatística devem ser realizados para confirmação dessa hipótese. 
ABSTRACT: Fecal incontinence is a disabling condition with relevant social costs. Many therapies are described. Objective: To evaluate the response to biofeedback and epidemiological profile, describing the used technique. Methods: A retrospective study in 3 years (June 2005 - June 2008). Results: Thirty patients, 26 women and 4 men, with an average age of 66. The number of normal pregnancies and births varied from none to six and hysterectomy was present in nine. Hypotonia in manometry was present in all patients. Eighteen patients were satisfied, ten were partially met, none was completely dissatisfied, and two have abandoned the therapy. Conclusion: The clinical therapy to biofeedback can be effective for incontinence, but the comprehension by patient appears to be the most important. Diabetes mellitus, anorectal surgery and hysterectomy were related to complaints of incontinence.

Keywords: biofeedback; fecal incontinence; exercise; manometry; quality of life.

\section{REFERÊNCIAS}

1. Byrne CM, Solomon MJ, Rex J, Young JM, Heggie D, Merlino C. Telephone vs face-to-face biofeedback for fecal incontinence: comparison of two techniques in 239 patients. Dis Colon Rectum 2005;48(12):2281-8.

2. Heymen S, Jones KR, Ringel Y, Scarlett Y, Whitehead WE. Biofeedback treatment of fecal incontinence. A critical review. Dis Colon Rectum 2001;44(5):728-36.

3. Terra MP, Dobben AC, Berghmans B, Deutekom M, Baeten CGMI, Janssen LWM, et al. Electrical stimulation and pelvic floor muscle training with biofeedback in patients with fecal incontinence: a cohort study of 281 patients. Dis Colon Rectum 2006;49(8):1149-59.

4. Solomon MJ, Pager CK, Rex J, Roberts R, Manning J. Randomized, controlled trial of biofeedback with anal manometry, transanal ultrasound, or pelvic floor retraining with digital guidance alone in the treatment of mild to moderate fecal incontinence. Dis Colon Rectum 2003;46(6):703-10.

5. Fynes MM, Marshall K, Cassidy M, Behan M, Walsh D, Phil D, et al. A prospective, randomized study comparing the effect of augmented biofeedback with sensory biofeedback alone on fecal incontinence after obstetric trauma. Dis Colon Rectum 1999;42(6):753-8.

6. Martínez-Puente MC, Pascual-Montero JA, García-Olmo D. Customized biofeedback therapy improves results in fecal incontinence. Int J Colorectal Dis 2004;19(3):210-4.

7. Peticca L, Pietroletti R, Ayabaca SM, Pescatori M. Combined biofeedback, physiotherapy and electrostimulation for fecal incontinence. Tech Coloproctol 2000;4:157-61.

8. Norton C, Kamm MA. Outcome of biofeedback for fecal incontinence. Br J Surg 1999;86(9):1159-63.

9. Enck P. Biofeedback training in disordered defecation: a critical review. Gig Dis Sci 1993;38(11):1953-60.

10. Chiarioni G, Scattolini C, Bonfante F, Vantini I. Liquid stool incontinence with severe urgency: anorectal function and effective biofeedback treatment. Gut 1993;34(11):1576-80.

11. Chiarioni G, Bassoti G, Stegagnini S, Vantini I, Whitehead WE. Sensory retraining is key to biofeedback therapy for formed stool fecal incontinence. Am J Gastroenterol 2002;97(1):109-17.

12. Leroi AM, Dorival MP, Lecouturier MF, Saiter C, Welter ML, Touchais JY, et al. Pudendal neuropathy and severity of incontinence but not presence of an anal sphincter defect may determine the response to biofeedback therapy in fecal incontinence. Dis Colon Rectum 1999;42(6):762-9.

13. Loening-Baucke V. Efficacy of biofeedback training in improviving fecal incontinence and anorectal physiologic function. Gut 1990;31(12):1395-402.

14. Miner PB, Donelly TC, Read NW. Investigation of the mode of action of biofeedback in treatment of fecal incontinence. Gig Dis Sci 1990;35(10):1291-8.

\section{Endereço para correspondência:}

André F. Accetta

Rua Mariz e Barros, 51, apto 1.201 - Icaraí

CEP: 24220-120 - Niterói (RJ), Brasil

E-mail: aaccetta@gmail.com 